\title{
Beiträge zur Kenntnis der Herzmuskelverfettungen.
}

\author{
Von \\ Dr. med. Pupko, \\ ehemaliger Medizinalpraktikant am pathol. Institut.
}

(Eingegangen am 25. März 1921.)

Die Verfettung der Herzmuskulatur wird jetzt anders gewertet wie früher, Während früher entsprechend der Virchowschen Lehre von der Fettmetamorphose der fettigen Degeneration des Herzens eine selbständige Stellung eingeräumt wurde, eine Auffassung, die von Rib b e r t und Kraus mit einigen Einschränkungen und Modifikationen immer noch vertreten wird, sind jetzt vie'e Autoren wie besonders Asch of $f$ und $\mathrm{Lu}$ bars ch, von den Klinikern Kre hl und Ro m b erg geneigt, der Verfettung der Herzmuskulatur eine sekundäre Stellung zuzuweisen. Sie fassen sie nicht als Ursache sondern als Folgəzustand von Herzaffektionen auf, in der Hauptsache als Folge von Stauungen, von entzündlichen Prozessen und von Störungen im Zellstoffwechsel aus äußeren und inneren toxischen Wirkungen und daraus resultierenden Zustandsänderungen des Protoplasmas, die eine Störung in der Verbrennung des aufgenommenen Fettes bedingen könnten oder ein Sichtbarwerden der in den Zellen ständig vorhandenen Lipoide (Borchers, Fettphanerose). Dementsprechend kommen experimentelle Arbeiten wie die von Hasenfeld und Fennywessy, Pal, Rodow zu dem Resultat, daß sehr stark verfettete Herzen noch eine gute Leistungsfähigkeit auch gegenüber starker Erhöhung der Anforderungen haben können, andere wie Wegelin und Arnold in ihren Mastversuchen kommen sogar zu dem Ergebnis, daß durch reichliche Ernährung eine physiologische Fettinfiltration des Herzens erreicht werden könne. Diese Ansicht deckt sich mit der Aschoffschen Auffassung, daß die Verfettung fetaler Herzen und des Hisschen Bündels im späteren Alter physiologisch ist. (Arbeit von Engel.) Aus diessen zum Teil widersprechenden Ansichten ergeben sich 3 Fragen, die sich nur an Hand eines großen Materials lösen lassen. Sie sind: I. Gibt es eine physiologische Verfettung der Herzmuskulatur oder wenigstens eines der funktionell verschiedenen Teile wie des Hisssschen Bündels? II. Verhalten sich überhaupt die funktionell verschiedenen Herzteile in bezug auf ihre Verfettung verschieden und ist das auf ihre eigenartige funktionelle Stellung zu beziehen? III. Ist 
die Verfettung des Herzmuskels für seine Leistungsfähigkeit schädlich?

Auf Veranlassung von Geh. Prof. Lubarsch habe ich zur Beantwortung dieser Fragen 100 Herzen untersucht, davon 10 fetale Herzen, 15 von $0-1$ J., 15 von $1-15$ J., von $15-25,25-35$ usw. je 12 Herzen, von 55-80 Jahren 12. Es wurden jedes mal Stücke aus dem r. und $l$. Ventrikel (Vorder- und Hinterwand, r. und 1. große Papillarmuskel, r. und 1. Vorhöfe, Herzohren, vom Hissschen Bündel der linke Schenkel und seine Endausbreitung in Formalin gehärtet, mit dem Gefriermikrostom geschnitten und mit Sudan Hämalaun gefärbt. Zur Beantwortung der Frage I müssen vor allem die Befunde an den fetalen Herzen herangezogen werden, da gerade bei diesen viele Autoren ein physiologisches Vorkommen von Fett behaupten. So sah Aschoff feinkörniges Fett in allen Organen Neugeborener, auch im Herzmuskel. Sehr reichlich Fett fand. er im Herzen eines Mäuseembryos. Er hält diese Vorgänge für physiologisch. Reinath sah in der Körpermuskulatur jüngerer Kalbsfeten sehr reichlich Fett, Hofba uer in 30 Herzen von bei der Geburt durch Asphyxie umgekommenen gesunden Kindern eine mehr oder weniger ausgedehnte feinkörnige Verfettung der Herzmuskulatur, ebenso bei 3 noch warm zur Sektion gekommenen Feten, wo also Autolyse völlig auszuschließen war. Er hält den Fettgehalt fetaler Herzen für physiologisch, durch günstige Ernährung, gleichmäßige Temperatur und geringe Bewegungsmöglichkeit bedingt. Im Gegensatz hierzu fand Eselein bei 48 Totgeburten nur 2 mal Fett im Herzmuskel. In 32 aus den Protokollen von 1919 und 1920 des Berliner path. Instituts ausgezogenen Fällen fand sich 9 mal mehr oder weniger Fett in den fetalen Herzmuskeln, 23 mal keines. Unter den 9 verfetteten Herzen stammen 2 von luetischen Frühgeburten, 2 von totgeborenen ausgetragenen Früchten (Kephalotrypsie Decapitation), bei einer ist als Todesursache Geburtstrauma angegeben.

Nun komme ich zu meinen eigenen Befunden. Die Untersuchungen wurden in der oben beschriebenen Art durchgeführt. Es handelte sich in allen Fällen um Früh- und Totgeburten, deren Organe soweit es sich feststellen läßt, keinen besonderen makroskopischen oder mikroskopischen pathologisch anatomischen Befund zeigten. In 5 Fällen, 32, 35, 40, 49, $35 \mathrm{~cm}$ langen Feten fand sich kein Fett, in 3 Fällen bei Totgeburten von 38 und $40 \mathrm{~cm}$ Länge und einer $35 \mathrm{~cm}$ langen Frühgeburt eine ausgedehnte feinkörnige fleckförmige Verfettung aller Herzteile, in 2 Fällen eine geringe fleckförmige feinkörnige Verfettung, also bei der sorgfältigsten Untersuchung nur in $50 \%$ der Fälle. Es ist danach anzunehmen, daß die Verfettung fetaler Herzmuskulatur nicht normal ist. Wäre sie es, so fänden wir sie sehr viel regelmäßiger, ganz abgesehen davon, daß es unmöglich ist, gerade für Herzen von menschlichen Feten pathologisehe 
Zustände völlig auszuschließen, da doch die Vorgänge die zu Frühgeburten und Aborten führen oder zur Tötung der reifen Frucht während der Geburt zwingen, an sich pathologisch sind.

Bei den übrigen 90 Herzen fand sich in 77 Fällen eine mehr oder weniger starke Verfettung von Herzmuskelfasern, die bis auf die wenigen Fälle von spurenweiser Verfettung stets eine deutliche pathologische Ursache hatten. In 55 Fällen war die Verfettung nur gering, $10 \mathrm{mal}$ mäßig, 12 mal stark, immer selbst bei den stärksten Verfettungen fleckförmig. Auf die einzelnen Altersstufen und verschiedenen Erkrankungen verteilte sich die Verfettung wie aus beiliegender Tabelle ersichtlich ist. Die stärksten und auch die mäßigen Verfettungen fanden sich fast nur bei akuten Infektionskrankheiten, 1 mal bei Eklampsie, 1 mal bei Urämie, in der Hauptsache bis zum 25. Lebensjahre. (18 von 22.) Bei chronischen Erkrankungen wie bei Lues und Tuberkulose, bei Carcinom, Arteriosklerose fand sich meist nur wenig Fett in den Herzmuskelfasern. Die stärkere Beteiligung des jugendlichen Alters an der Verfettung erklärt sich wohl weniger aus dem Altersunterschied als aus der größeren Disposition für Infektionskrankheiten. Daß mit dem Alter die Häufigkeit der Fälle abnimmt, in denen das Herz völlig fettfrei ist, die Zahl der Fälle mit geringer Verfettung zunimmt (von 13 fettfreien. Herzen fallen 9 auf das 1.-25. J., von 55 Herzen mit geringer Verfettung 29 auf das 35.-75. J.) ist auf die häufigere chronische Insuffizienz mit Stauungszuständen zurückzuführen. Außerdem fand ich in Ưbereinstimmung mit Schlüter, der häufig eine besonders starke Fettinfiltration in der Umgebung von Schwielen sah, ein Befund, den er auf örtliche Lymphstauung zurückführt, sehr häufig besonders in der Umgebung von Schwielen Verfettung in kleinen atrophischen Muskelfasern. Von 10 Herzen der Altersstufe 55-75 J. boten 7 diesen Befund. Die geringen scheinbar von der Schwielenbildung unabhängigen Verfettungen könnten in diesen Füllen z. T. wenigstens auf durch Schwielen bedingte lokale Stauungen, in der Umgebung der Schwielen gleichfalls auf Stauungen und auf unmittelbare Schädigung der Muskelfasern selbst zurückzuführen sein. Gerade atrophische kleine von Bindegewebszügen unterbrochene Fasern sind verfettet. Wir sehen hier eine deutliche Trennung in Verfettungen aus lokalen Ursachen und Verfettungen als Folge von Allgemeinerkrankungen, letztere bei jüngeren Individuen vor allem bei akuten Infektionskrankheiten, erstere bei älteren Leuten, Schwielenbildungen infolge von Sklerose oder abgelaufener entzündlicher Zustände mit nachfolgenden lokalen Stauungen. Ist so, was wohl auch niemand bestreitet, die Verfettung des Herzmuskels im allgemeinen eine auf verschiedenen. Ursachen beruhende durchaus krankhafte Erscheinung sekundärer Natur, bleibt noch die Frage offen, ob nicht doch in einzelnen funktionell deutlich isoliert dastehenden Teilen eine physiologische 
Verfettung vorkommen kann und ob überhaupt ein Unterschied in der Verfettung einzelner Teile nachweisbar ist. Eine isolierte Verfettung einzelner Herzteile fand sich in 15 von 98 Fällen. Sie verteilte sich auf die einzelnen Herzabschnitte wie folgt:

Hisssches Bündel 2 mal,

linker Papillarmuskel $1 \mathrm{mal}$,

rechtes Herzohr $1 \mathrm{mal}$.

Eine auf Vorhöfe H. B. und einen Papillarmuskel beschränkte Verfettung 11 mal. Auf einer deutlichen lokalen Ursache beruhte die Verfettung nur $1 \mathrm{mal}$, in einem Falle von Meaortitis luetica, wo im 1. Papillarmuskel starke fast diffuse Schwielenbildung, Blutungen und eine ausgedehnte feinkörnige Verfettungen zu finden waren, während das übrige Herz keine Besonderheiten zeigte. Worauf die Verfettung in den anderen Fällen beruhte, läßt sich nicht ohne weiteres sagen. Nur beim 1. Papillarmuskel ließe sich ein Zusammenhang zwischen seiner funktionellen Inanspruchnahme und der Verfettung finden. Da die Verfettung am häufigsten und am stärksten an der Spitze zu finden war, besonders in atrophischen Fasern, so wäre es denkbar, daß sie hier dieselben Ursachen haben könnte, die zu der so häufigen fibrösen Atrophie führen. (Im linken Papillarmuskel fand ich sie in 22 Fällen, davon 20 mal kombiniert mit einer mehr oder weniger starken Verfettung von vor allem subendocardial und an der Spitze gelegenen Muskelfasern.) Daß die Verfettung des H.B. und der Vorhöfe mit ihren stärkeren Fettgewebsum- und Durchwachsungen zusammenhängt, ist nicht anzunehmen. Dann müßte sie in den Vorhöfen und vor allem in den Herzohren sehr viel häufiger sein. Vor allem steht damit imWiderspruch, daß bei allgemeiner gleichmäßiger Fettgewebsdurchwachsung des Herzens die Verfettung von Herzmuskelfasern sehr ungleichmäßig, mitunter sehr gering ist. Es müssen hier also andere unbekannte Faktoren mit im Spiele sein. Hier wäre noch der Befund von Borchers in Betracht zu ziehen, der bei seinen Untersuchungen über die Fettphanerose einen stärkeren Lipoidgehalt des H. B. und des linken Papillarmuskels fand. Es wäre also möglich, daß geringe, vielleicht noch in den Grenzen des Physiologischen liegende Zustandsänderungen des Sarkoplasmas gerade in den lipoidreichsten Teilen des Herzens ein Sichtbarwerden von Lipoiden bedingen könnten. Für die Beurteilung dieser Zustände ist es noch von gewissem Interesse, daß sich unter diesen isolierten Verfettungen 2 Herzen von Selbstmördern fanden. Bei einem sah ich eine geringe feinkörnige Verfettung einzelner Muskelfasern im H. B., linken Vorhof, linken und rechten Herzohr, beim anderen eine geringe feinkörnige Verfettung im linken und rechten Papillarmuskel. Ein dritter Selbstmörder hatte sogar eine geringe feinkörnige Verfettung in allen Herzteilen. In allen 3 Fällen war keine Fettembolie vorhanden und auch sonst keine pathologischen Veränderungen zu finden außer dem Lokal- 
befund (Kopfschüsse). Wenn man auch trotz der negativen Befunde nicht sicher sagen kann, daß̣ es sich hier um völlig normale Individuen handelt, ein Selbstmord ist schließlich an und für sich nicht normal, so ist es hier doch unmöglich zu entscheiden, ob es sich nicht doch noch um in Grenzen des Physiologischen liegende Vorgänge handelt. Im Gegensatz zu meinen Befunden sah Eselein in 11 Unglücksfällen kein Fett im Herzmuskel, doch hat er nicht so zahlreiche und verschiedene Abschnitte des Herzens untersucht. Engel dagegen fand bei einem durch Unfall umgekommenen Potator eine ausgedehnte isolierte Verfettung des H. B. Auch in Wegelins 2 Fällen von raschem Tod nach. Unfall bei sonst gesunden Individuen war eine ausgedehnte feinkörnige Verfettung des Herzmuskels vorhanden.

Wir haben hier álso eine isolierte Verfettung einzelner Herzteile gefunden, die aber im ganzen nicht sehr häufig ist und deren Ursachen z. T. wenigstens unklar sind. Es wäre noch zu prüfen, ob diese Herzteile auch bei allgemeiner Verfettung der Herzmuskulatur ein abweichendes Verhalten zeigen. Für das Hisssche Bündel liegen in dieser Hinsicht bereits eine Reihe von Untersuchungen vor. Ein genaues Literatur; erzeichnis findet sich in dem Aufsatz von Mönck eberg im Lubarsch Ostertag (Jahrg. XIX).

Die meisten Autoren schließen sich der Ansicht von Lubarsch und Aschoff an, daß der H. B. zwar besonders häufig verfettet, daß aker seiner isolierten Verfettung keine völlig selbständige Bedeutung in pathologisch-anatomischer Hinsicht zukommt, sondern daß sie nur durch seine Lage im lockern subendocardialen Bindegewebe und durch seine selbständige Gefäßversorgung bedingt ist, über die Bedeutung und die Folgen der Verfettung des H. B., daß eine Funktionsstörung nur dann denkbar ist, wenn die Verfettung sehr hochgradig und mit entzündlichen und degenerativen Prozessen kombiniext ist, also mehre eine Folgeerscheinung der übrigen Veränderungen, nicht der Verfettung.

Was meine eigenen Befunde anbetrifft, so sah ich nach der von L ubarsch gegebenen Einteilung:

1. Völliges Freibleiben der Schenkel bei mäßiger herdförmiger Verfettung $14 \mathrm{mal}$.

2. Geringe Verfettung des H. B. bei starker herdförmiger Verfettung der Triebmuskulatur 1 mal.

3. Isolierte Verfettung der Bündelfasern 2 mal.

4. Erheblich stärkere Verfettung der Bündelfase:n bei geringer herdförmiger Verfettung der Triebmuskulatur 11 mal.

5. Gleichmäßige Verfettung von H. B. und Triebmuskulatur $47 \mathrm{mal}$.

Eine Zunahme der Verfettungstendenz der Bündelfasern mit dem Alter wie Engel sie fand und Asch off sie für pbysiologisch hält, konnte ich nicht feststellen. Es fehlte jede deutliche Beziehung zwischen Ver- 
fettung des H. B. und Alter. Dagegen sah ich auch in meinen Fällen Schwielen und entzündliche Prozesse besonders häufig und besonders stark im H. B. und im umgebenden Bindegewebe.

Auch der linke Papillarmuskel und die Vorhöfe zeigen eine wenn auch etwas geringere Selbständigkeit in der Verfettung.

Es fand sich:

1. Völliges Freibleiben der Papillarmuskeln bei mäßiger Verfettung der Triebmuskulatur $10 \mathrm{mal}$.

2. Isolierte Verfettung des linken Papillarmuskels 1 mal.

3. Erheblich stärkere Verfettung des linken Papil]armuskels bei Verfettung des Myocards geringeren Grades 8 mal.

4. Gleichmäßige Verfettung $58 \mathrm{mal}$.

Fibröse Atrophie des linken großen Papillarmuskels fand sich $22 \mathrm{mal}$, des rechten $10 \mathrm{mal}$. Beziehungen zwischen Verfettung und Alter bestanden hier ebensowenig wie im H. B. Bemerkenswerte Unterschiede in der Verfettung des rechten und linken Herzens fanden sich nicht. Kurz zusammengefaßt ist also die Frage, ob sich die funktionell verschiedenen Herzteile in bezug auf ihre Verfettung verschieden verhalten, dahin zu beantworten, daß ein wirklich deutliches Abweichen im Verhalten gegenüber der übrigen Muskulatur nur dem H. B. und linken Papillarmuskel zukommt, nur beim linken Papillarmuskel vielleicht infolge seiner besonderen funktionellen Inanspruchnahme, beim Hissschen Bündel wahrscheinlich auf Grund seiner topographisch-anatomischen Verhältnisse. Die Verfettung der einzelnen Herzteile ist im übrigen so sprunghaft wechselnd, daß wir eine große Gesetzmäßigkeit bisher nicht zu finden vermögen. Diese Mannigfaltigkeit ist wohl durch die Verschiedenartigkeit der Faktoren bedingt, die zur Verfettung führen und die infolge der verschiedenartigen anatomischen und vielleicht auch funktionellen Beziehungen der einzelnen Herzteile zueinander nicht überall gleichmäßig da sind. Daß die Verfettung einzelner Herzteile physiologisch ist, kann schon deshalb nicht angenommen werden, weil sie dann mit viel größerer Regelmäßigkeit zu finden sein müßte. Jedoch besteht die Möglichkeit, daß ganz geringe Verfettungen bei sonst gesunden Individuen durch noch im Bereiche des Normalen liegende Stoffwechselschwankungen bedingt sein könnten. Was nun die Folgen der Herzverfettung anlangt, so sind die Beziehungen zwischen Herzschwäche und Herzverfettung viel zu unklar, als daß sich hier etwas Sicheres sagen ließe. Jedenfalls müßten, wenn Herzschwäche und Herzmuskelverfettung in unmittelbarem Zusammenhang stünden, sehr viel mehr Herzen und sehr viel ausgedehnter verfettet gefunden werden, da doch in den meisten Fällen die letzte Todesursache Herzinsuffizienz ist. 


\section{Literaturverzeichnis.}

1) Ribbert, Verhandl. d. D. path. Gesellschaft 1903, VI. Tg. - ${ }^{2}$ ) Kra us, Fettige Degeneration des Herzmuskels. Berl. klin. Wochenschr. 1905, Nr. 442. 3) Aschoff, Über die nenern anatomischen Befunde am Herzen und ihre Beziehungen zur Herzschwäche. Med. Klinik 1909, Nr. 8 u. 9. - ${ }^{4}$ ) Aschoff und Taw ara, Lehre von den pathologisch-anatomischen Grundlagen der Herzschwäche. Jena 1906. - ${ }^{5}$ ) Aschoff, Herzstörungen und ihre Beziehungen zum speziellen Muskelsystem des Herzens. $-{ }^{6}$ ) Asch off, Fettgehalt fötaler Gewebe. Zentralbl. f. allg. Pathol. u. pathol. Anat. 8. 1897. - 7) Lubars ch, Herzpathologie, insbesondere pathologische Anatomie der Herzschwäche. Jahresk. f. ärztl. Fortbild. 1911. - ${ }^{8}$ ) Krehl, Erkrankungen des Herzmuskels. Wien 1901 (im Nothnagel). $\left.{ }^{9}\right)$ Krehl, Beitrag zur Pathologie der Herzklappenfehler. Dtsch. Arch. f. klin. Med. 46, 1890. - 10) Ro mberg, Über die Erkrankungen des Herzmuskels bei Typhus abd., Scharlach und Diphtherie. Dtsch. Arch. f. klin. Med. 48 u. 49. - ${ }^{11}$ ) Hasen feld und Fennywessy, Über die Leistungsfähigkeit des fettig entarteten Herzens. Berl. klin. Wochenschr. 1899, Nr. 4, 6 u. $7 .-{ }^{12}$ ) Pal, Bedeutung der Herzmuskelveränderungen bei Phosphorvergiftung. Zeitschr. f. Heilk. 1900. 13) Rodow, Die Wirkung des Phosphors auf die Herzmuskulatur. Zeitschr. f. klin. Med. 34. 1912. - ${ }^{14}$ ) Wegelin, Über alimentäre Herzverfettung. Berl. klin. Wochenschr. Nr. 46 u. 47. 1913. - ${ }^{15}$ ) Arnold, Fettumsatz und Fettwanderung. Virchows Archiv 179. 1903. - ${ }^{16}$ ) Engel, Beiträge zur normalen und pathologischen Histologie des Atrioventri.-Bündels. Zieglers Beiträge z. allg. Pathol. u. pathol. Anat. 48. - ${ }^{17}$ ) Reinath, Über den mikroskopischen Nachweis von Fett im normalen Muskel. Inaug.-Diss. Freiburg 1909. - ${ }^{18}$ ) Hof bauer, Physiologischer Fettgehalt fötaler Herzen. Anat. Anz. 27. 1905. - ${ }^{19}$ ) Eselein, Untersuchungen über den Fettgehalt der Herzmuskulatur. Virchows Archiv 218. 1914. - ${ }^{20}$ ) Schlüter, Die Frlahmung der hypertropischen Herzmuskulatur. Leipzig u. Wien 1906. - ${ }^{21}$ ) B orchers, Die Rolle der Fettphanerese bei den krankhaften Veränderungen der Herzmuskulatur. Virchows Archiv 218. 